\title{
Architecture Design and FPGA Implementation of an FFT based Reactive Power Meter
}

\author{
MSR Naidu \\ Assoc.Prof., Dep. of ECE, \\ AITAM, Tekkali, India
}

\author{
G R Locharla \\ Asst.Prof., Dept. of ECE \\ GMRIT, Rajam, India
}

\author{
GBSR Naidu \\ Asst.Prof., Dept. of ECE \\ GMRIT, Rajam, India
}

\begin{abstract}
Reactive power measurement is increasingly paid attention by power industry. A novel architecture to measure the reactive power is proposed in this paper. Architecture is described in verilog and implemented using Xilinx ISE 13.1i. In this method voltage and current signals in time domain are converted to the frequency domain and power on each component is calculated to obtain the total reactive power.
\end{abstract}

\section{Keywords}

Xilinx, FFT, FPGA, DFF

\section{INTRODUCTION}

Novel methods of Reactive power measurement are drawing the attention of modern power system engineers due to the challenges in providing accuracy. In this modern era of electronic technology, power grids have been added to the large number of non-linear loads. This is resulting in a lot of harmonics into the power grids, causing serious distortion in power system voltage, current. Harmonic pollution will not only increase the wear and tear of the power components, but endanger the safe operation of power systems. Where sinusoidal variations of the power supply are not pure and multiple harmonics of fundamental frequency is present, electrical equipment like motors will not work properly and it leads to poor power factor, so that electrical equipment will not be utilized to their maximum capability. This also leads to wear and tear of transmission line. Therefore a system that measures the harmonic distortion plays an important role in the feedback system of the power grid in providing quality. By the architecture proposed in this paper, power is calculated through converting time domain voltage and current signals in to the frequency domain and summing the power on frequency component[6]. Low value for the relative error of the reactive power measured by FPGA is obtained, which is around $0.4 \%$. Still this can be drastically minimized by using floating point multipliers inside the architecture implementation instead of fixed point multipliers. Architecture implementation is done using Xilinx ISE 13.1i and XC3S1200eFG320 device of Spartan3E family is targeted.

\section{PROPOSED METHOD}

The formula of reactive power for the sine wave can be shown as Equation 1.

$$
P=\operatorname{Irms}^{*} \operatorname{Vrms}^{*} \sin \theta
$$

The relationship between the maximum and the RMS value for a sine wave can be given by Equation (2)

$$
V_{r m s}=V_{m} / \sqrt{2}, I_{r m s}=I m / \sqrt{2}
$$

Equation (2) is substituted into Equation1, then we can get the Equation (3).

$$
P_{k}=1 / 2 * I_{r m s}{ }^{*} V_{r m s}{ }^{*} \sin \theta
$$

In the formula (3) Im and Um are all maximums. For number $\mathrm{k}$ harmonic in the

non- sinusoidal condition, the formula of reactive power can be expressed as (4):

$$
P_{k}=1 / 2 * I_{m}(k) * V_{m}(k) * \sin \theta_{K}
$$

Then the total reactive power[7] is shown in Equation(5).

$$
\text { Ptotal }=\sum_{k} 1 / 2 * I_{m}\left(\begin{array}{ll}
k & *
\end{array} V_{m}\left(\begin{array}{ll}
k & *
\end{array}\right) \sin \theta_{K} \ldots . .\right. \text { (5) }
$$

For number $\mathrm{k}$ harmonic, we assume $\mathrm{x} 1$ and $\mathrm{y} 1$ representing voltage transform results. $\mathrm{X} 1$ and $\mathrm{y} 1$ are the results of FFT core. $\mathrm{X} 1$ is real part, $\mathrm{y} 1$ is imaginary part. In the same way, $\mathrm{x} 2$ and $\mathrm{y} 2$ are current transform results. $\mathrm{X} 2$ and $\mathrm{y} 2$ are results of FFT core. $\mathrm{X} 2$ is the real part, $\mathrm{y} 2$ is the imaginary part. The result of calculation through FFT cannot be used directly to compute the maximum value of voltages and currents and we need to scale the data. The scaling is done in the following manner, the outputs of FFT should be divided by 64 and then multiply with 2 . Dividing by $2^{6}$ is because of accumulation effect of fft and multiplying 2 is because the relationship of sine function and exponential function

$$
\cos (x)=\left(e^{x}+e^{-j x}\right) / 2
$$

The scaled values are

$$
\left.\begin{array}{l}
X_{1}^{\prime}=2 * X_{1} / 2^{6} \\
Y_{1}^{\prime}=2 * X_{1} / 2^{6} \\
X_{2}^{\prime}=2 * X_{2} / 2^{6} \\
Y_{2}^{\prime}=2 * X_{2} / 2^{6}
\end{array}\right\}
$$

The magnitude maximums can be expressed as

$$
\left.\begin{array}{l}
V_{m}(k)=\sqrt{X_{1}^{\prime 2}+Y_{1}^{\prime 2}} \\
I m \\
I^{(k)}=\sqrt{{X_{2}^{\prime 2}+Y_{2}^{\prime 2}}^{2}}
\end{array}\right\}
$$

For number $\mathrm{k}$ harmonic, $\theta \mathrm{u}$ is the voltage phase, $\theta \mathrm{v}$ is the current phase

$$
\left.\begin{array}{r}
\sin \theta_{u}=Y_{1} / \sqrt{X_{1}^{2}+Y_{1}^{2}} \\
\cos \theta_{u}=X_{1} / \sqrt{X_{1}^{2}+Y_{1}^{2}} \\
\sin \theta_{i}=Y_{2} / \sqrt{X_{2}^{2}+Y_{2}^{2}} \\
\cos \theta_{i}=X_{2} / \sqrt{X_{2}^{2}+Y_{2}^{2}}
\end{array}\right\}
$$


The formulae for phase seperation can be expressed as $\sin \theta_{k}=\sin \left(\theta_{u}-\theta_{i}\right)$

$$
=\sin \theta_{u} \cos \theta_{i}-\cos \theta_{u} \sin \theta_{i}
$$

The power of a particular harmonic is given by:

$$
P_{K}=\left(Y_{1} * X_{2}-X_{1} * Y_{2}\right) / 2^{11}
$$

The total harmonic power is given by:

$$
P=\sum_{K=1}^{N} P_{K}
$$

\section{FPGA IMPLEMENTATION}

The proposed system module contains: Two FFT cores, Two multipliers subtraction module, Transposing module, Accumulator module to calculate the equation (12).

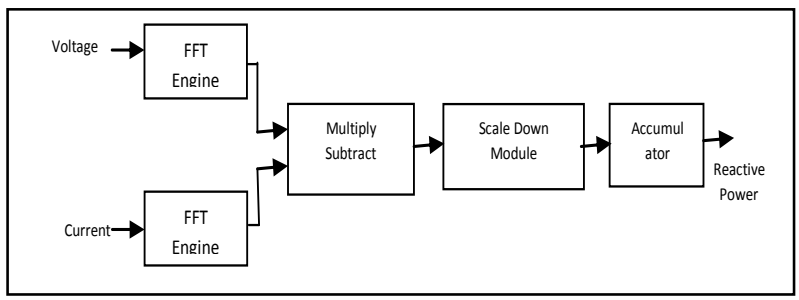

Fig 3.1 Block Diagram Of The Power Meter

\subsection{FFT Engine}

Two FFT cores are used to estimate the magnitudes and phases of each harmonic for voltage and current inputs.

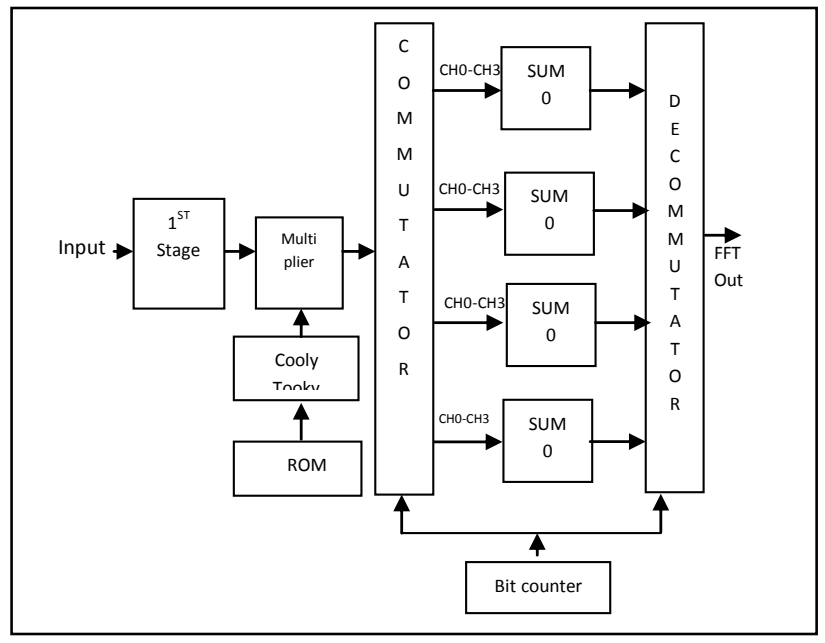

3.2 FFT Engine Architecture

Initially FFT architecture consists of RST, CLK AND EN pins which are given as inputs to the FFT block. The reset pin performs initialization and termination operations. The clock signal will synchronize the entire operation.

\subsection{Multiply Subtract unit}

Multiply subtract unit calculates the numerator of the eq (11).,

\subsection{Scale down module}

Scale down unit performs the division in eq(11)This used minimize the bus width which holds the $\mathrm{k}^{\text {th }}$ power component.

\subsection{Accumulator}

This module is used to add the reactive power of all harmonics. This unit calculates the eq(12).

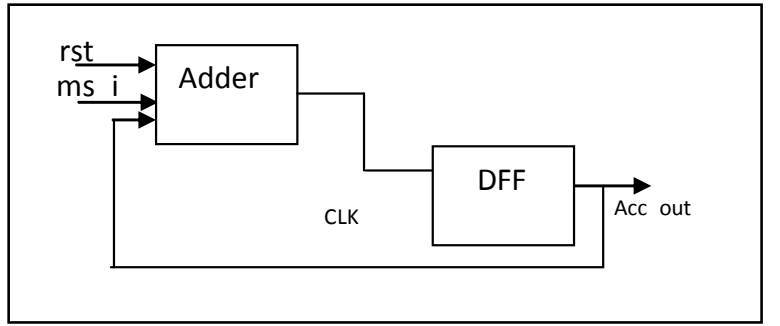

Fig 3.3 Accumulator

\section{RESULTS}

\subsection{Simulation Results}

4.1.1 Interface signals of FFT unit (voltage)

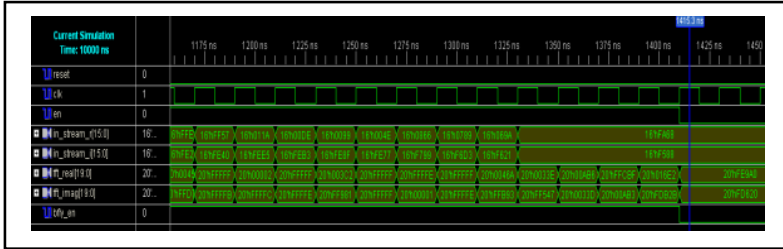

Fig 4.1 FFT Waveforms of Voltage

\subsubsection{Interface signals of FFT unit (current)}

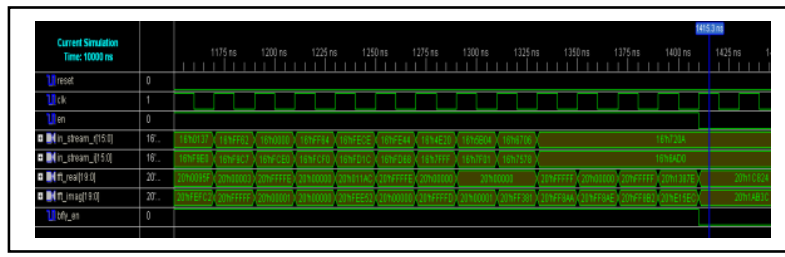

Fig 4.3 FFT Waveforms of Current

\subsubsection{Interface signals of multiply subtract unit}

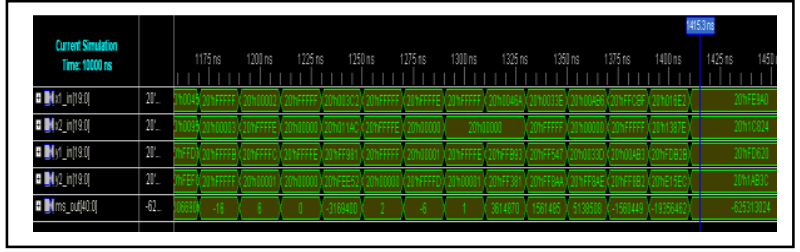

Fig 4.1 Multiply Subtract Unit- Waveforms

\subsubsection{Interface signals of Accumulator unit}

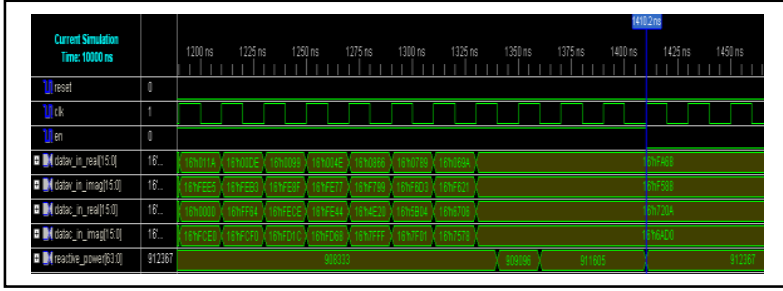

Fig 4.4 Reactive Power Result 


\subsection{Synthesis Results}

\subsubsection{RTL Schematic: In Out Pins}

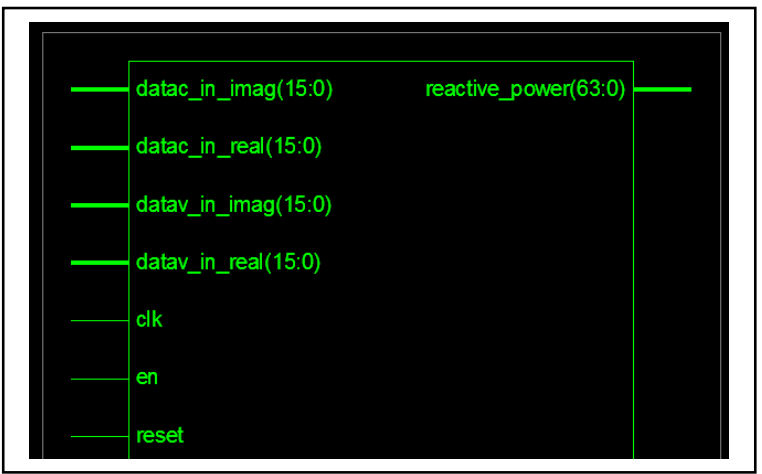

Fig 4.5 Top module's Port layout

\subsubsection{RTL Schematic: Top Module}

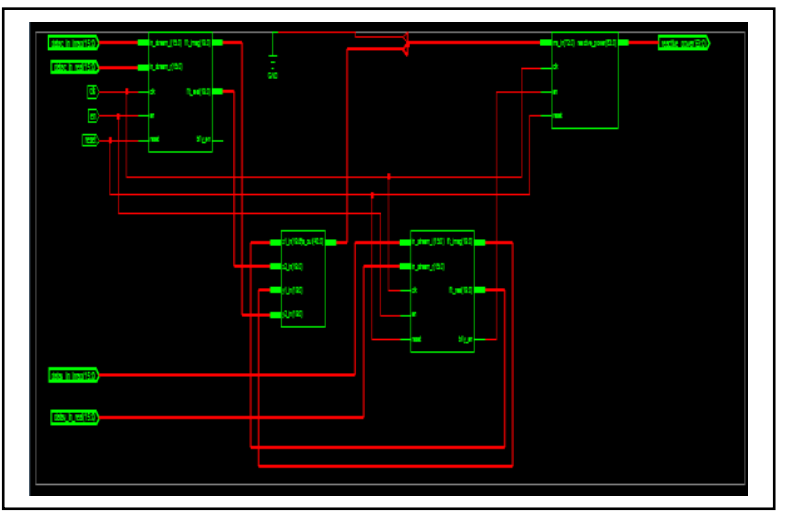

Fig 4.6 Top module

\subsubsection{RTL Schematic: FFT Engine}

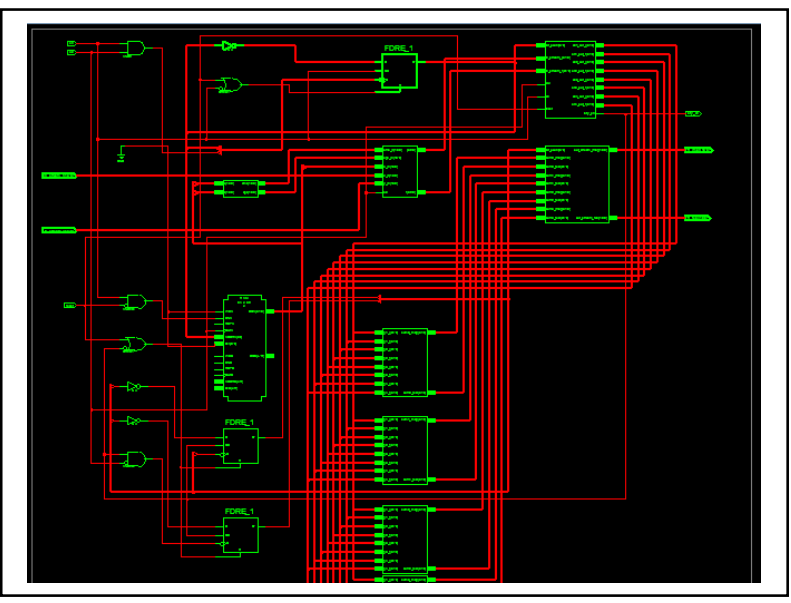

Fig 4.7 FFT Engine

\subsection{Device Utilization Summery}

FPGA synthesis by targeting Spartan 3e (XILINX) device results the requirement of FPGA components as seen in the Table.1.
Table 1. Device Utilization Summery

\begin{tabular}{|c|l|c|}
\hline S. No. & FPGA Resource & Utilization \\
\hline 1 & Slices & 6487 \\
\hline 2 & Slice Flip Flops & 548 \\
\hline 3 & 4 input LUTs & 1237 \\
\hline 4 & Ios & 131 \\
\hline 5 & Bonded IOBs & 131 \\
\hline 6 & BRAMs & 2 \\
\hline 7 & GCLKs & 10 \\
\hline
\end{tabular}

\section{PERFORMANCE}

Reactive power using 'MATLAB' $=916310$

Reactive power using 'FPGA' $=912367$

The relative error of the simulation effect and data in theory is $=((916310-912367) / 916310) * 100 \%$ $=0.4303 \%$

\section{CONCLUSION}

Architecture is implemented in Verilog and Xc3s1200e$4 \mathrm{fg} 320$ device of Spartan 3E family is targeted. FPGA simulation result obtained is very close to the theoretical result from Matlab. So it is preferable to develop an ASIC with this architecture to achieve highly accurate reactive power measurement.

\section{REFERENCES}

[1] S. A. Soliman, R. A. Alammari M. E. El-Hawary, "Effects of Harmonic Distortion on the Active and Reactive Power Measurements in theTime Domain: A Single Phase System." 2001 IEEE Porto Power Tech Conference

[2] Leszek, s. Czarnecki, "Measurement of the Individual Harmonics Reactive Power in Nonsinusoidal Systems", IEEE Transactions On Instrumentation And Measurement.

[3] Jun Rongi, Gui-xin Zhangi "Electric Energy easurement in Digital Substation on A Number of Issues Discussed", CICED2008

[4] Branislav Djokic', Senior Member, IEEE, "A High Performance Frequency Insensitive Quadrature Phase Shifter and Its Application in Reactive Power Measurements", IEEE Transactions On Instrumentation And Measurement.

[5] Elham B. Makram, Senior Member, IEEE "Effect of Harmonic Distortion in Reactive Power Measurement", IEEE Transactions on Industry Applications, vol. 28, no. 4, july / august 1992.

[6] Adalet N. Abiyev, Kamil Dimililer "Reactive Power Measurement in Sinusoidal and Nonsinusoidal Conditions by Use of the Walsh Functions", I2MTC 2008 - IEEE International Instrumentation and Measurement Technology Conference, Victoria, Vancouver Island, Canada, May 12-15, 2008

[7]AN-758, Application Note, Analog Devices, One Technology Way, P.O. Box 9106, Norwood, MA 02062-9106,Tel: 781/329-4700, Fax: 781/461-3113 www.analog.com 